University of Nebraska - Lincoln

DigitalCommons@University of Nebraska - Lincoln

3-1989

\title{
Differential Cross Sections for Ejection of Electrons from Rare Gases by 7.5- 150-keV Protons
}

\author{
Wen-qin Cheng \\ University of Nebraska - Lincoln \\ M. Eugene Rudd \\ University of Nebraska - Lincoln, erudd@unl.edu \\ Ying-Yuan Hsu \\ University of Nebraska - Lincoln
}

Follow this and additional works at: https://digitalcommons.unl.edu/physicsrudd

Part of the Physics Commons

Cheng, Wen-qin; Rudd, M. Eugene; and Hsu, Ying-Yuan, "Differential Cross Sections for Ejection of Electrons from Rare Gases by 7.5- 150-keV Protons" (1989). M. Eugene Rudd Publications. 3.

https://digitalcommons.unl.edu/physicsrudd/3

This Article is brought to you for free and open access by the Research Papers in Physics and Astronomy at DigitalCommons@University of Nebraska - Lincoln. It has been accepted for inclusion in M. Eugene Rudd Publications by an authorized administrator of DigitalCommons@University of Nebraska - Lincoln. 


\title{
Differential cross sections for ejection of electrons from rare gases by $7.5-150-k e V$ protons
}

\author{
Wen-qin Cheng, ${ }^{*}$ M. E. Rudd, and Ying-Yuan Hsu \\ Behlen Laboratory of Physics, University of Nebraska, Lincoln, Nebraska 68588-0111
}

(Received 26 July 1988)

\begin{abstract}
Absolute cross sections for the ejection of electrons by proton impact that are differential in the angle and energy of the electrons have been measured for helium, neon, and krypton. The primary energy ranged from 7.5 to $150 \mathrm{keV}$, the angles from $10^{\circ}$ to $160^{\circ}$, and the measured energies from 1 to $550 \mathrm{eV}$. These data along with earlier data on argon are used to study the systematics of protonimpact ionization of the rare gases. An analytical model is employed to correlate and systematize the cross-section differential in ejected-electron energy. For low-energy collisions the angular distribution of electrons ejected into the forward hemisphere is found to be independent of the electron energy and largely independent of the target. The Massey adiabatic criterion yields results for the energy of the maxima of the electron-ejection functions, in disagreement with experiment. Doubly differential cross sections for neon calculated from the distorted-wave Born approximation show only fair agreement with experimental data. A method of determining electron multiplier efficiency based on Poisson statistics is described.
\end{abstract}

\section{INTRODUCTION}

Although the ejection of electrons in collisions accounts for the greater part of the energy loss of ions passing through matter, there are no theoretical methods or combination of methods for calculation which give accurate, detailed information about the distribution of cross sections with electron energy and ejection angle for all targets and energies. The Born approximation is useful only for the simplest targets and only at high collision energies. The classical binary-encounter approximation and the Monte Carlo method also lose accuracy when the incident particle velocity becomes smaller than that of the orbital electron in the target.

Lacking an $a b$ initio method of calculation, investigators have sought an understanding of the systematics of electron ejection through semiempirical models or other generalizations from experimental data. At the higher energies (above about 50 or $100 \mathrm{keV}$ ) several studies have been made of the doubly differential cross sections (DDCS) for this process. Toburen, Manson, and $\mathrm{Kim}^{1}$ investigated electron ejection from rare-gas targets by protons up to $5 \mathrm{MeV}$ by plotting the ratio $Y(E, T)$ of the measured singly differential cross sections (SDCS) to the Rutherford cross section per electron as a function of the electron energy. Such a plot can be compared to photoionization data to obtain information, e.g., on the numbers of target electrons involved in the ionization process and on oscillator strengths. As they point out, this method of study of atoms also applies to molecules as targets since there is no explicit dependence on the wave function, only on the inner- and outer-shell binding energies of the targets.

Miller, Toburen, and Manson ${ }^{2}$ and Wilson, Miller, and Tobure $^{3}$ have developed a model, based on Bethe's theory, giving the SDCS as a function of the ejectedelectron energy. This model, too, it useful only at high energies.

A study of the systematics of the doubly differential cross sections for this process was made by Crooks and
Rudd $^{4}$ and by Rudd, Gregoire, and Crooks ${ }^{5}$ for proton energies of 50-300 keV. They found, e.g., that while a simple scaling by numbers of active electrons sufficed to relate the DDCS for nitrogen and oxygen targets, this scaling did not work when comparing neon and helium.

There are few studies of the systematics of the electron-ejection process at low energies. Rudd and Madison $^{6}$ presented data and calculations for helium bombarded by $5-100-\mathrm{keV}$ protons. While Born approximation calculations using Hartree-Fock wave functions followed the general dependences of the DDCS on angle and ejection energy to unexpectedly low energies, discrepancies of considerable magnitude remained.

$\mathrm{Rudd}^{7,8}$ has presented a semiempirical model based on the classical binary-encounter approximation and the Bethe theory at large proton and small electron energies and on the molecular promotion model at small proton and large electron energies. In this model a single equation with two adjustable parameters which fits the singly differential cross sections at all electron energies may be used over the entire range of proton energies.

In this paper we present DDCS and, by integration, SDCS for helium, neon, and krypton targets for $7.5-150-\mathrm{keV}$ proton collisions. No previous data for krypton exist at these energies and data have been published on neon only at $50 \mathrm{keV}$ and above. ${ }^{1,4}$ Measurements on helium were made to compare with earlier data. $^{6}$ When the present results are combined with existing data on argon, ${ }^{9}$ a comparison may be made among four of the rare gases. The semiempirical $\operatorname{model}^{8}$ is used to fit the present data.

\section{EXPERIMENTAL METHOD}

\section{A. Apparatus}

The apparatus and techniques used in this experiment were similar to those of earlier work ${ }^{6,10}$ so only a brief description will be given. A magnetically analyzed proton beam was finely collimated before entering the col- 
lision region and was caught by a biased, shielded Faraday cup. The pressure of the target gas, which was typically $0.5 \mathrm{mT}$ Torr, was measured by a capacitance manometer. Electrons ejected along a short length of the beam path were selected by a slit system with an angular resolution of $1.4^{\circ}$. They were then accelerated by $5 \mathrm{~V}$ before entering a parallel-plate electrostatic analyzer with a full width at half maximum (FWHM) resolution of $5.5 \%$. The detector was an 18-stage venetian-blind-type electron multiplier with the first dynode biased at $80 \mathrm{~V}$. Three pairs of Helmholtz coils were used to null out magnetic fields. The collision region and the system of slits selecting the secondary electrons were in a field-free region and the geometry was such that all of the electrons of the proper energy entering the analyzer should reach the detector. Therefore the transmission efficiency of the analyzer was nominally $100 \%$. However, because of stray fields, this efficiency may have been smaller for electrons with energies less than about $10 \mathrm{eV}$. Since these fields varied in an unpredictable way, it was not possible to correct for their effect.

\section{B. Detection efficiency}

The overall detection efficiency was the product of the electron multiplier efficiency $\eta_{m}$ and the fraction $f_{d}$ of pulses passed by the discriminator. The value of $\eta_{m}$ was determined to be $0.81 \pm 0.08$ by a procedure described in the Appendix. To obtain the value of $f_{d}$, an integral pulse-height distribution was plotted and extrapolated to zero pulse height. The count rate at the discriminator setting used divided by the extrapolated value gave $0.85 \pm 0.04$ for $f_{d}$. These values along with measured geometrical quantities, collected beam charge, and target densities allowed us to calculate absolute values of the cross sections.

\section{Uncertainties}

Most of the $16 \%$ basic uncertainty in the cross sections resulted from a $12 \%$ uncertainty in the pressure measurement and a $10 \%$ uncertainty in the detector efficiency. In addition, there are the usual difficulties of transporting low-energy electrons which cause data in that range to be less accurate. At the lower proton energies the beam currents were small and sometimes erratic, causing additional errors. Statistical counting errors and uncertainties in background subtraction were generally negligible unless the cross sections were smaller than about $10^{-27}-10^{-26} \mathrm{~m}^{2} / \mathrm{eV} \mathrm{sr}$.

\section{EXPERIMENTAL RESULTS}

\section{A. Singly differential cross sections}

The integration over angles indicated by the equation

$$
\sigma(W)=2 \pi \int_{0}^{\pi} \sigma(W, \theta) \sin \theta d \theta
$$

was done numerically for each electron energy $W$ giving the SDCS. The results for helium are compared in Fig. 1 to earlier data from our laboratory ${ }^{6}$ and with recent data

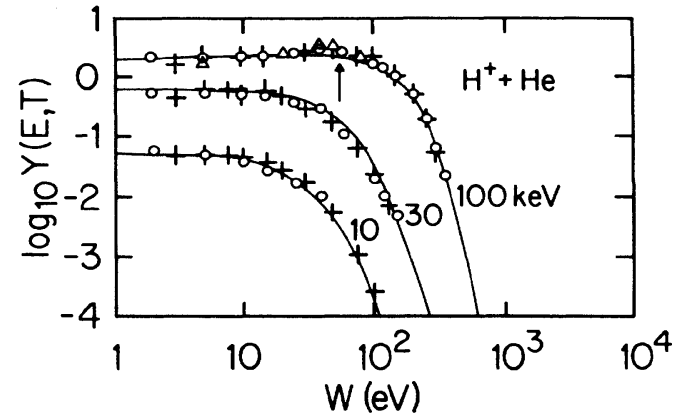

FIG. 1. Energy distributions of electrons from $\mathrm{H}^{+}+\mathrm{He}$ collisions. Circles, present data; crosses, data of Rudd and Madison (Ref. 6); triangles, data of Gibson and Reid (Ref. 11); lines, Eqs. (1) and (2). Vertical arrow shows expected position of the electron transfer to the continuum peak for $100 \mathrm{keV} . Y(E, T)$ is the ratio of the measured cross section to the Rutherford cross section per electron.

by Gibson and Reid. ${ }^{11}$ For each set of data, the lowenergy SDCS were adjusted according to the procedure described by $\mathrm{Rudd}^{8}$ to yield the recommended total cross sections. ${ }^{12}$ The data by Rudd and Madison ${ }^{6}$ required an especially large adjustment but the final result was in good agreement with the other two sets of data. The vertical arrow on the graph indicates the expected position of the peak due to electron transfer to the continuum. ${ }^{13}$ This peak appears in the $100-\mathrm{keV}$ data but is too small to be noticeable in the SDCS for the 30- and 10-keV data. The cross sections are given as ratios to the Rutherford cross section per electron, a method of presentation suggested by $\mathrm{Kim}$ and Inokuti ${ }^{14}$ to reduce the wide range of values plotted and to facilitate further analysis. The quantity

$$
Y(E, T)=T E^{2} \sigma(W) / 4 \pi a_{0}^{2} R^{2}
$$

is plotted where $T=m v_{p}^{2} / 2, m$ is the electron mass, $v_{p}$ the proton velocity, $a_{0}$ the Bohr radius, and $R$ the Rydberg energy. $E$ is the energy transfer given by $E=W+I_{1}$ where $I_{1}$ is the binding energy of the outermost shell.

The neon SDCS data are compared in the same fashion in Fig. 2 to earlier data by Crooks and Rudd. ${ }^{4}$ The agreement between these two sets of data is also quite satisfactory. Figure 3 shows some of the krypton data.

A model for the SDCS has been developed ${ }^{7,8}$ which yields the equation

$$
\begin{aligned}
\sigma(W)= & (S / I)\left(F_{1}+F_{2} w\right)(1+w)^{-3} \\
& \times\left[1+\exp \left(\alpha\left(w-w_{c}\right) / v\right)\right]^{-1},
\end{aligned}
$$

where $w=W / I, I$ is the binding energy of the electron in a given shell of the target, $v=(T / I)^{1 / 2}$ is the reduced projectile velocity, $S=4 \pi a_{0}^{2} N(R / I)^{2}$, and $w_{c}$ is the cutoff energy given by $w_{c}=4 v^{2}-2 v-R / 4 I . N$ is the number of electrons in a given shell of the target atom. $F_{1}$ and $F_{2}$ are the two adjustable fitting parameters and $\alpha$ is a dimensionless constant which is slightly different for different targets. $F_{1}$ and $F_{2}$ are functions only of $v$ and, when chosen properly, the cross section reduces to that given by the Bethe theory in the limit of high impact en- 


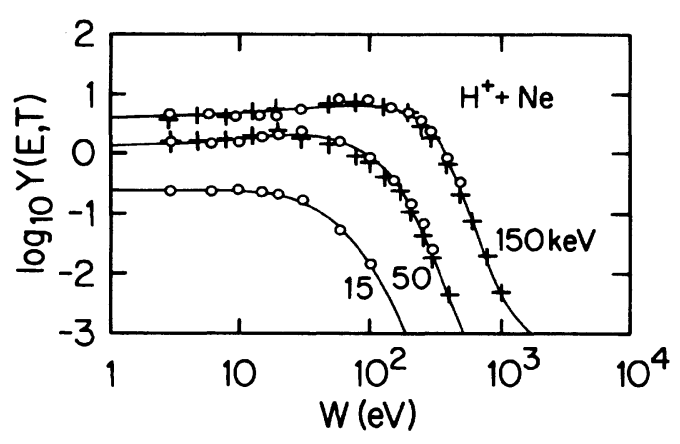

FIG. 2. Energy distributions of electrons from $\mathbf{H}^{+}+\mathrm{Ne}$ collisions. Circles, present data; crosses, data of Crooks and Rudd (Ref. 4); lines, Eqs. (1) and (2).

ergies. The equations used to fit $F_{1}$ and $F_{2}$ were

$$
F_{1}=L_{1}+H_{1}, \quad F_{2}=L_{2} H_{2} /\left(L_{2}+H_{2}\right),
$$

where

$$
\begin{aligned}
& L_{1}=C_{1} v^{D_{1}} /\left(1+E_{1} v^{\left(D_{1}+4\right)}, \quad L_{2}=C_{2} v^{D_{2}},\right. \\
& H_{1}=A_{1} \ln \left(1+v^{2}\right) /\left(v^{2}+B_{1} / v^{2}\right), \\
& H_{2}=A_{2} / v^{2}+B_{2} / v^{4} .
\end{aligned}
$$

The quantities $A_{1}, \ldots, E_{1}$ and $A_{2}, \ldots, D_{2}$ and $\alpha$ are the basic fitting parameters which give the SDCS for all incident and ejection energies. When enough data are available to determine the parameters, the contribution to the SDCS may be calculated for each shell and the results added to obtain the measured SDCS. The values of $N$ and $I$ for the various shells are known from other experiments and calculations. ${ }^{15}$ Values for the targets considered here are given in Table I.

By including higher-energy data of other investigators, ${ }^{1,9,16}$ the curves of $F_{1}$ and $F_{2}$ were determined over the entire range of proton energies available. These are shown in Fig. 4 and the parameters fitting these curves in Table II. In fitting the model to the data the condition was imposed that the integral of the SDCS over electron energy had to yield the recommended total cross section as given in the review paper by Rudd et al. ${ }^{12}$ The calculated cross sections from Eqs. (1) and (2) using the param-

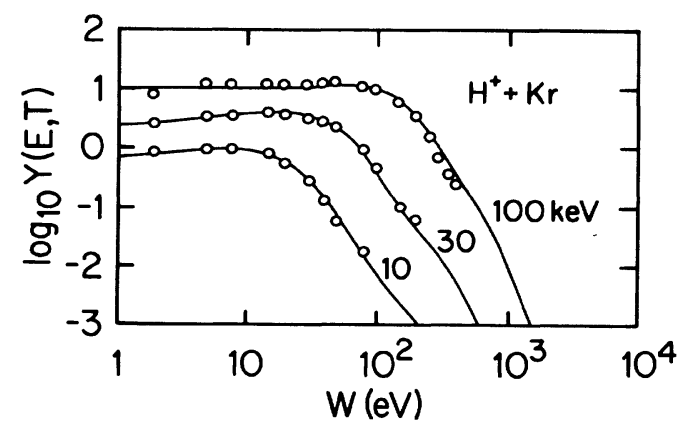

FIG. 3. Energy distributions of electrons from $\mathrm{H}^{+}+\mathrm{Kr}$ collisions. Circles, present data; lines, Eqs. (1) and (2).
TABLE I. Numbers of electrons and binding energies for outer shells of four target gases.

\begin{tabular}{lccc}
\hline \hline Target & Shell & $N$ & $I(\mathrm{eV})$ \\
\hline Helium & $1 s$ & 2 & 24.6 \\
Neon & $2 p$ & 6 & 21.6 \\
& $2 s$ & 2 & 48.5 \\
Argon & $3 p$ & 6 & 15.8 \\
& $3 s$ & 2 & 29.2 \\
& $2 p$ & 6 & 249 \\
& $2 s$ & 2 & 326 \\
Krypton & $4 p$ & 6 & 14.3 \\
& $4 s$ & 2 & 27.5 \\
& $3 d$ & 10 & 94.5 \\
& $3 p$ & 6 & 217 \\
& $3 s$ & 2 & 292 \\
\hline \hline
\end{tabular}

eters of Tables I and II are shown as lines in Figs. 1-3. The agreement between the experimental data and the model is very good. The effect of the inner shells is evident at the high-energy end of the curves for krypton.

The SDCS for particular ejected-electron energies are plotted against the proton energy in Fig. 5 for neon. Data of Crooks and Rudd ${ }^{4}$ and of Toburen, Manson, and $\mathrm{Kim}^{1}$ have been used to extend the range of the curves. If a log-log plot of the energy at the maximum of these curves is made against the energy transfer $E=W+I$, the result is a nearly straight line with a slope of $0.71 \pm 0.04$. This is at variance with the Massey adiabatic criterion which predicts a slope of 2 . Evidently this criterion does not apply to electron ejection.

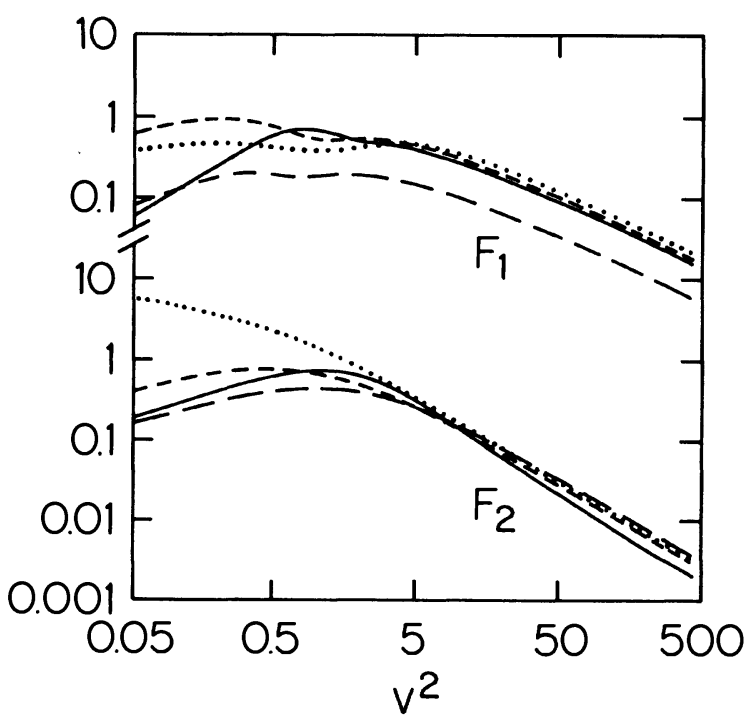

FIG. 4. Values of the dimensionless fitting parameters $F_{1}$ and $F_{2}$ for the four gases as a function of the square of the reduced impact velocity. Solid line, helium; long-dashed line, neon; short-dashed line, argon; dotted line, krypton. 
TABLE II. Values of parameters for Eq. (2).

\begin{tabular}{lllll}
\hline & $\mathrm{He}$ & $\mathrm{Ne}$ & $\mathrm{Ar}$ & $\mathrm{Kr}$ \\
\hline$A_{1}$ & 1.00 & 0.36 & 1.10 & 1.29 \\
$B_{1}$ & 3.3 & 0.82 & 1.50 & 4.6 \\
$C_{1}$ & 1.31 & 0.53 & 1.84 & 0.57 \\
$D_{1}$ & 2.1 & 1.28 & 0.78 & 0.30 \\
$E_{1}$ & 1.92 & 14.6 & 8.1 & 2.4 \\
$A_{2}$ & 0.84 & 1.53 & 1.20 & 1.37 \\
$B_{2}$ & 5.0 & 0.0 & 0.10 & 3.1 \\
$C_{2}$ & 0.84 & 0.64 & 1.15 & 1.86 \\
$D_{2}$ & 1.04 & 0.90 & 0.74 & -0.70 \\
$\alpha$ & 0.86 & 0.70 & 0.72 & 0.79 \\
\hline \hline
\end{tabular}

\section{B. Doubly differential cross sections}

Since the SDCS are well described by Eqs. (1) and (2), the DDCS may be referred to them by giving the ratio $f(\theta)=\sigma(W, \theta) / \sigma(W)$. This ratio is plotted versus $W$ for each angle for the four rare gases at $100 \mathrm{keV}$ in Fig. 6. Data on argon from Crooks and Rudd ${ }^{4}$ have been included for comparison among the targets. To a first approximation the curves are horizontal straight lines indicating that the angular distribution is the same for all secondary-electron energies. The major exceptions are at small angles (for which the electron transfer to the continuum peaks shows up at $54 \mathrm{eV}$ and the binaryencounter humps at $220 \mathrm{eV}$ are most visible) and at large angles (where the Auger peaks in argon at $200 \mathrm{eV}$ are most noticeable).

\section{Variation of angular distribution with ejected electron energy}

For small incident proton energies there is remarkably little variation in the angular distributions with electron

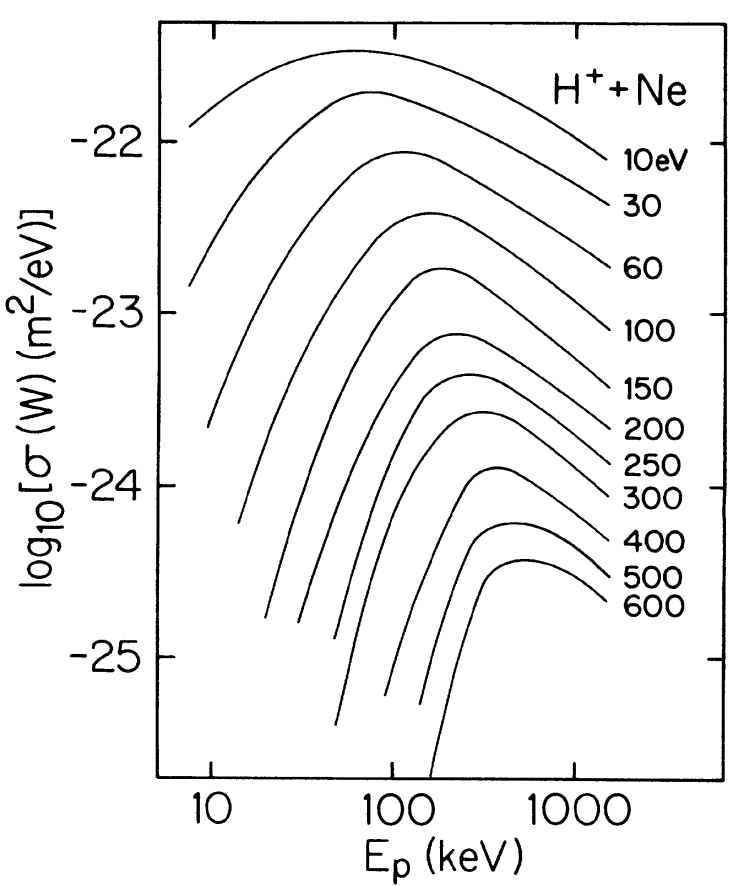

FIG. 5. SDCS for neon vs proton energy for electron-ejection energies from 10 to $600 \mathrm{eV}$.

energy. Figure 7 shows that the $7.5-30-\mathrm{keV}$ data for krypton follow a nearly universal curve as the secondary energy is varied. Except for a somewhat greater variation in the backward directions, the data generally fall within a $\pm 25 \%$ range. However, for $70 \mathrm{keV}$ and to a greater extent for $150 \mathrm{keV}$ there is a larger variation. This is caused by the emergence of the binary-encounter peak at the higher energies. This peak comes at a
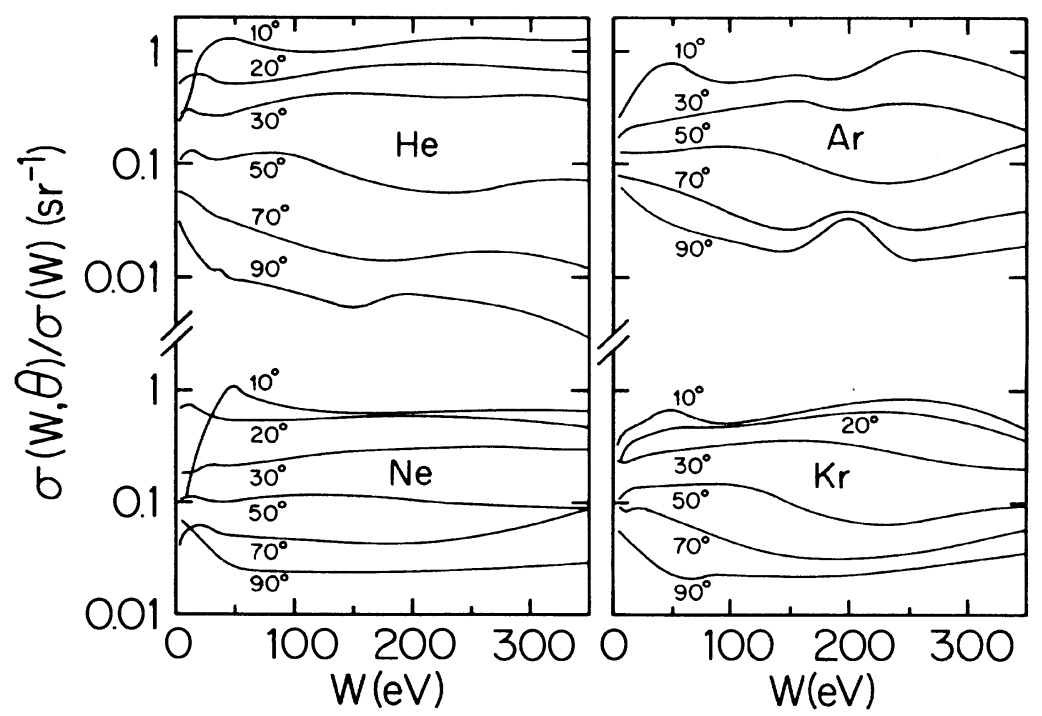

FIG. 6. Ejected-electron energy distributions for various angles, normalized to integrated values for four targets. 


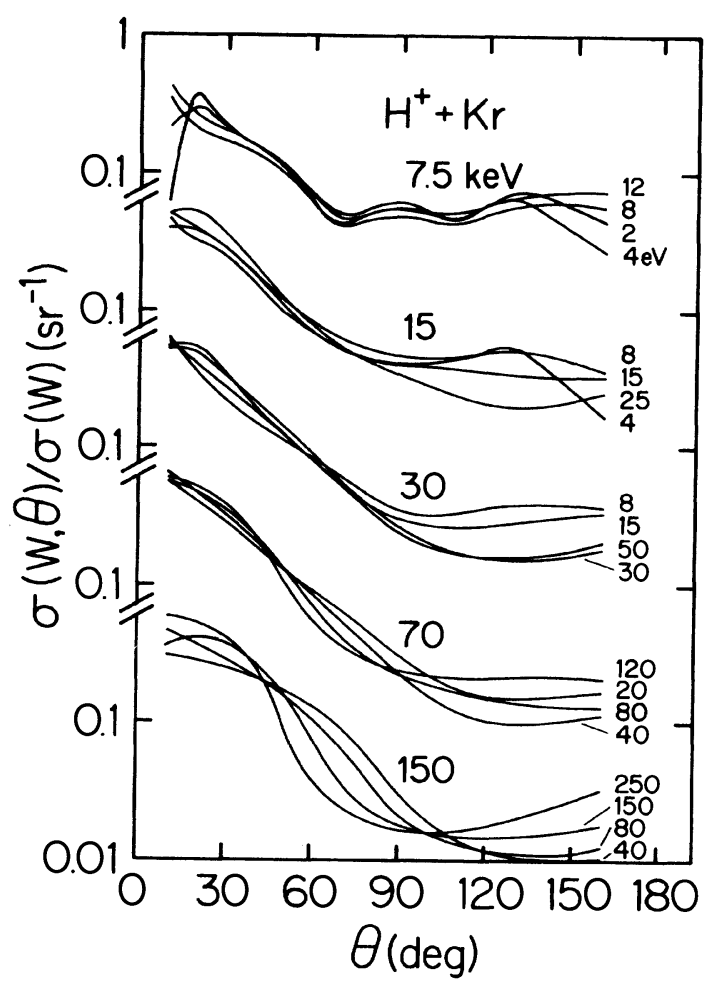

FIG. 7. Effect of electron-ejection energy on the angular distributions of electrons for various proton energies for krypton. For each proton energy the electron energies were approximately $W=0.5 T, T, 2 T$, and $3 T$.

different angle for different electron energies and therefore affects the shape of the angular distribution.

\section{Variation of angular distribution with target gas}

Figure 8 shows that except for the backward hemisphere, there is little variation in the angular distributions at small impact energies among the four target gases. Within a $\pm 40 \%$ variation, the values of $f(\theta)$ follow universal curves. Except for a slightly greater anisotropy for helium, there seems to be no systematic variation among the targets. There is often an increase in the cross sections with angle above $90^{\circ}$, the largest rise appearing for neon and argon targets.

\section{Variation of angular distribution with impact energy}

Figure 9 shows data at various impact energies with the electron energies chosen at $W=2 T$. The angular distributions become more isotropic the smaller the proton energy, a behavior which is also evident if data at different proton energies are compared at the same electron energies. This change is very pronounced for helium, less so for neon and argon, and is nearly absent for krypton.

\section{Comparison with theory}

Madison $^{6,17}$ has applied the distorted-wave Born approximation (DWBA) to proton collisions using wave

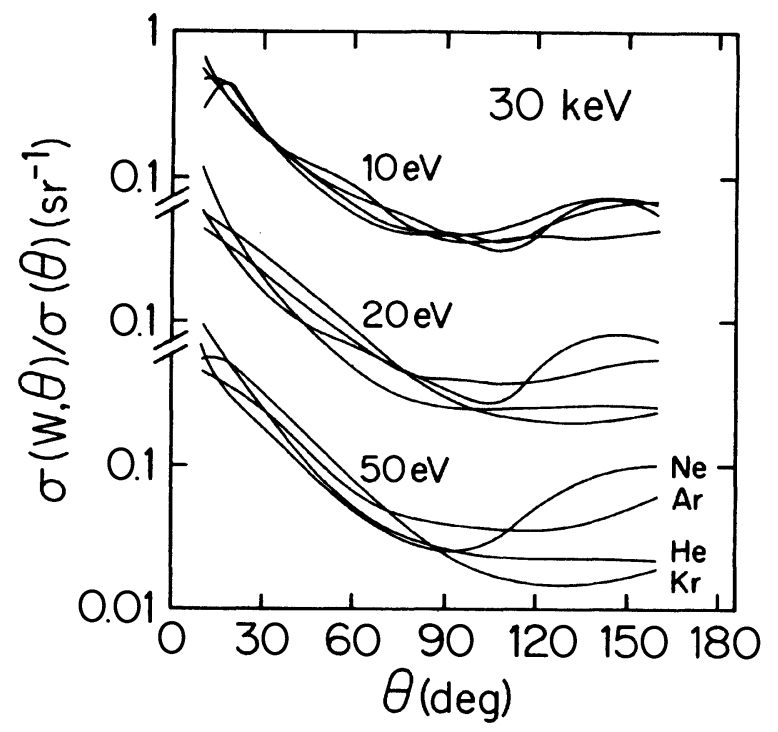

FIG. 8. Effect of target species on the angular distributions of electrons of various energies produced by $30-\mathrm{keV}$ proton collisions for four gases.

functions derived from a Hartree-Fock potential. Integrating the triply differential cross sections over the momentum transfer $q$, DDCS are obtained which may be compared with those measured in this project. It has been shown ${ }^{6}$ that the use of more realistic wave functions improves the agreement over what is obtained with

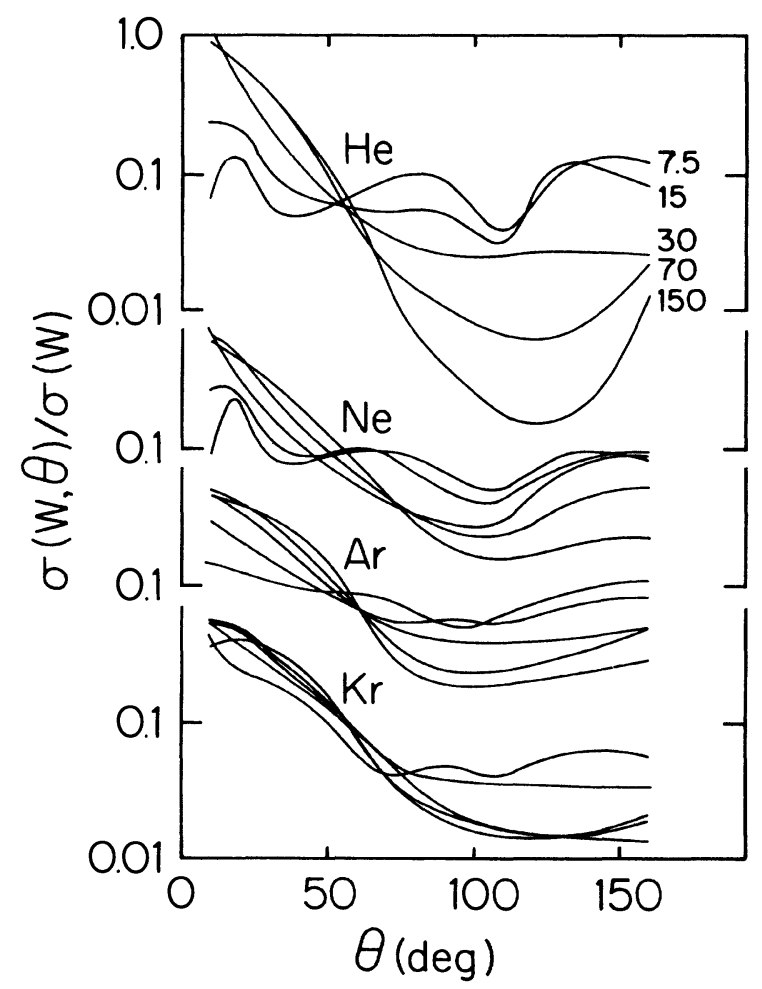

FIG. 9. Angular distributions of electrons ejected from four targets by $7.5-150-\mathrm{keV}$ proton collisions. In each case the electron energy was $W=2 T$. 


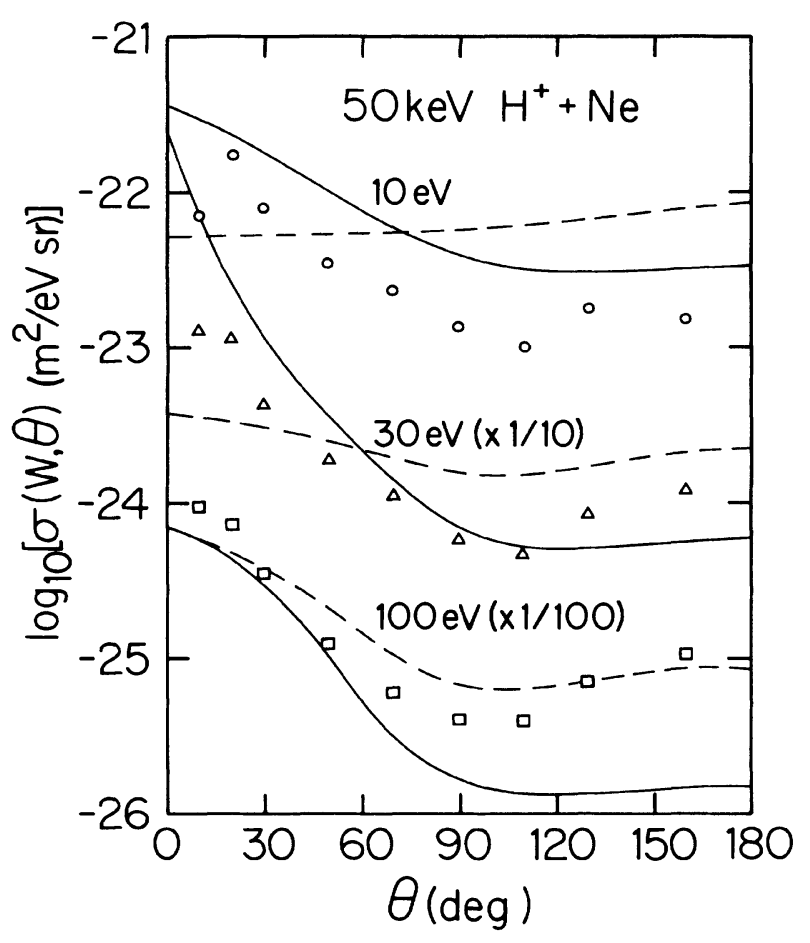

FIG. 10. Angular distribution of electrons of $10-100-\mathrm{eV}$ energy from $50-\mathrm{keV} \mathrm{H}^{+}+\mathrm{Ne}$ collisions. Circles, triangles, and squares are present data; dashed lines, DWBA calculations; solid lines, DWBA calculations with Salin factor.

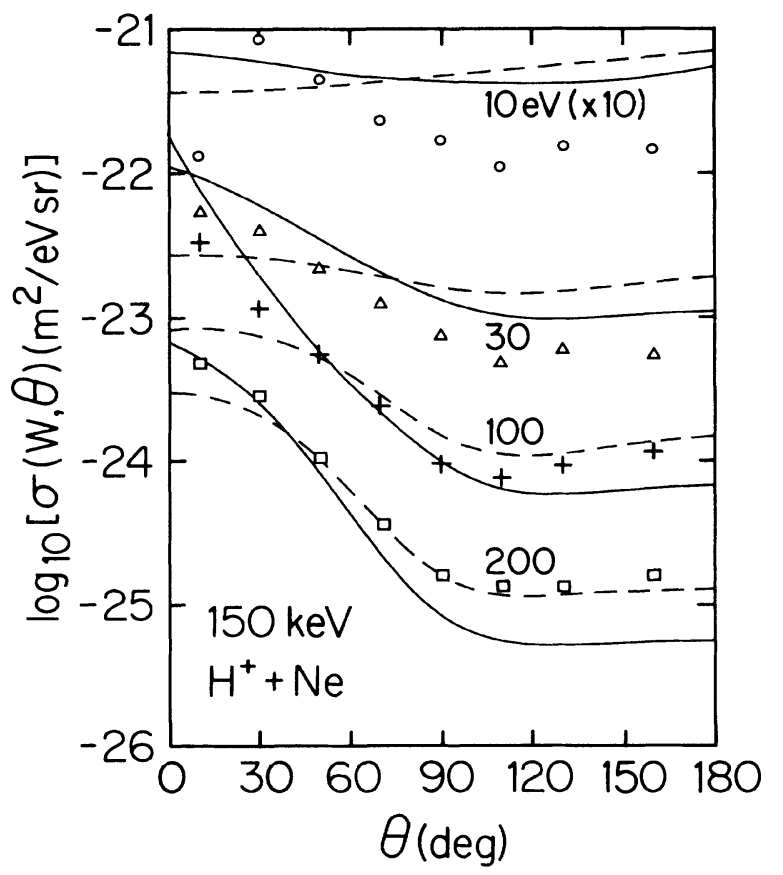

FIG. 11. Angular distribution of electrons of $10-200-\mathrm{eV}$ energy from $150-\mathrm{keV} \mathrm{H}^{+}+\mathrm{Ne}$ collisions. Legend as in Fig. 10. scaled hydrogenic wave functions. Madison ${ }^{18}$ has calculated DDCS for the $2 s$ and $2 p$ subshells of neon, the sum of which should be a good approximation to the present data since electrons in the $1 s$ shell are too tightly bound to contribute appreciably to the cross sections. To take account of the effect of the electron transfer to the continuum process, Salin ${ }^{19}$ has suggested a multiplicative factor be applied to the DDCS.

Figures 10 and 11 show the comparison between the data and the DWBA with and without the Salin factor. The agreement varies from poor at low electron and low proton energies to fairly good at high proton and electron energies. The Salin factor improves the agreement at small angles in most cases but usually results in poorer agreement at large angles.

\section{CONCLUSIONS}

It has been shown that the model given in Eqs. (1) and (2) describes the SDCS well even for low incident proton energy collisions. By making adjustments in the measured cross sections such that the integrated values correspond to recommended values for the total cross sections, a consistent set of differential cross sections is obtained over a wide range of incident and secondary energies which are specified by the values of a set of ten basic parameters for each target. These parameters for helium, neon, argon, and krypton are given in Table II.

The angular distributions, specified by the ratio $f(\theta)=\sigma(W, \theta) / \sigma(W)$, were studied as the incident energy, the secondary-electron energy, and the target species were varied. At low incident energies (energies for which $T<I)$ it is found that in the forward hemisphere $f(\theta)$ is independent of the ejected-electron energy and shows only small nonsystematic variations among the different targets. $f(\theta)$ generally has a larger rise at the backward angles for neon and argon than for helium or krypton. Changing the incident proton energy has a large effect on $f(\theta)$ for helium, but a smaller influence for the other gases. For krypton $f(\theta)$ in the forward hemisphere is nearly independent of incident energy.

A comparison between the measured DDCS for neon and those given by the DWBA indicates that although the general trends of the data are roughly reproduced by the theory, discrepancies of factors of 3 are not uncommon even at the highest energy $(150 \mathrm{keV})$. Adding the Salin factor to account for electron transfer to the continuum generally improves the agreement at small angles but worsens it at large angles.

The Massey adiabatic criterion for the position of the maximum in the cross section as a function of incident energy fails when applied to the ejection of electrons of various energies.

A method is described to determine the detection efficiency of an electron multiplier from a measurement of the integral pulse-height distribution. This method assumes that the production of electrons at each dynode follows Poisson statistics. That this is a good assumption is shown by the good agreement between predicted and measured pulse-height distributions. 


\section{ACKNOWLEDGMENTS}

The authors wish to thank D. Madison for supplying the DWBA calculations for neon and D. K. Gibson for providing his experimental data in tabular form. This paper is based on work supported by National Science Foundation Grants No. PHY-8401328 and No. PHY8701905.

\section{APPENDIX}

The method used to determine the efficiency of the electron multiplier detector assumes that the number of secondary electrons per incident primary electron at each stage of multiplication follows a Poisson distribution. Then, assuming that none is lost between stages, one may calculate the probability of the multiplier producing no electrons at the last $(K)$ stage when one electron strikes the first stage. If this probability is $P_{K}(0)$ then $1-P_{K}(0)$ is the detection efficiency. The probability $P_{K}(0)$ is a function only of the average gain per stage $\epsilon$, which is the basic quantity to be measured in this method. This can be done by measuring the integral pulse-height distribution (PHD) and the gain of the preamplifier-amplifier combination used. These are relatively simple measurements compared to the more cumbersome methods usually used to determine absolute efficiencies of detectors.

Lombard and Martin ${ }^{20}$ have derived the necessary equations to calculate the expected PHD and the efficiency as a function of $\epsilon$. For a single stage the probability of producing $n$ secondary electrons per incident primary is given by the Poisson distribution

$$
P_{1}(n)=\epsilon^{n} e^{-\epsilon} / n ! \text {. }
$$

The probability of $n$ electrons appearing after $K$ stages is obtained by iterating the equation

$$
\begin{aligned}
& P_{K}(n)=(\epsilon / n) \sum_{i=0}^{n-1}(n-i) P_{K}(i) P_{K-1}(n-i), \\
& n \neq 0, \quad K>1 .
\end{aligned}
$$

For the special case of $n=0$,

$$
P_{K}(0)=\exp \left\{-\epsilon\left[1-P_{K-1}(0)\right]\right\} .
$$

While Eq. (A3) may be readily iterated, the computer time required to iterate Eq. (A2) goes up rapidly with the number of stages. But since the shape of the PHD curve is largely determined by the first 4 to 8 stages (depending on the gain per stage), it is not necessary to iterate over all stages.

In their paper Lombard and Martin indicated that their multipliers (with unspecified dynode material) did not follow the PHD predicted by Eq. (A2). Baldwin and Friedman, ${ }^{21}$ using a multiplier with $\mathrm{Ag}-\mathrm{Mg}$ dynodes, obtained exponential PHD's also in disagreement with those predicted by Poisson statistics. However, Tusting, Kerns, and Knudsen ${ }^{22}$ found that their photomultipiers did yield Poisson-type PHD's. We also find Poisson PHD's for the electron multiplier used in the present work. This was an 18-stage venetian-blind-type multiplier with $\mathrm{Cu}-\mathrm{Be}-\mathrm{O}$ dynodes (EMI type 9462/4B). For

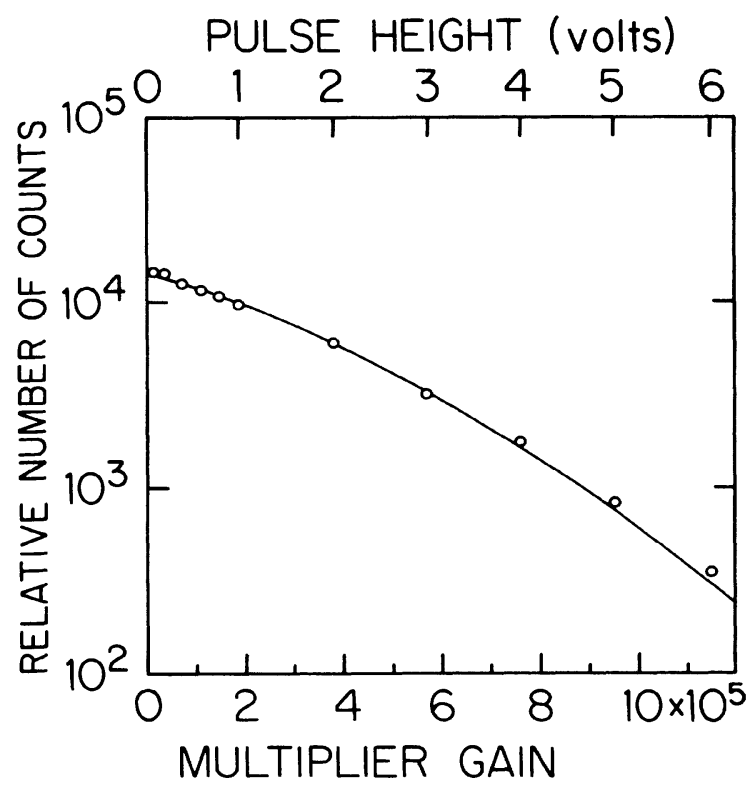

FIG. 12. Pulse-height distribution from the 18-stage electron multiplier used in this work. Top scale, pulse height in volts; bottom scale, pulse height divided by the transfer gain of the amplifier to obtain the multiplier gain. The line gives values calculated from Eq. (A2).

the measurement of the PHD 300-eV electrons were directed to the first dynode which was at $80 \mathrm{~V}$ above ground. The last dynode was at $2800 \mathrm{~V}$ and a resistor string distributed the potential equally among the remaining dynodes.

The points plotted in Fig. 12 represent the measured integral PHD. The top scale is the discriminator setting and therefore the minimum size of pulses counted. Using the measured transfer gain of the preamplifier-amplifier

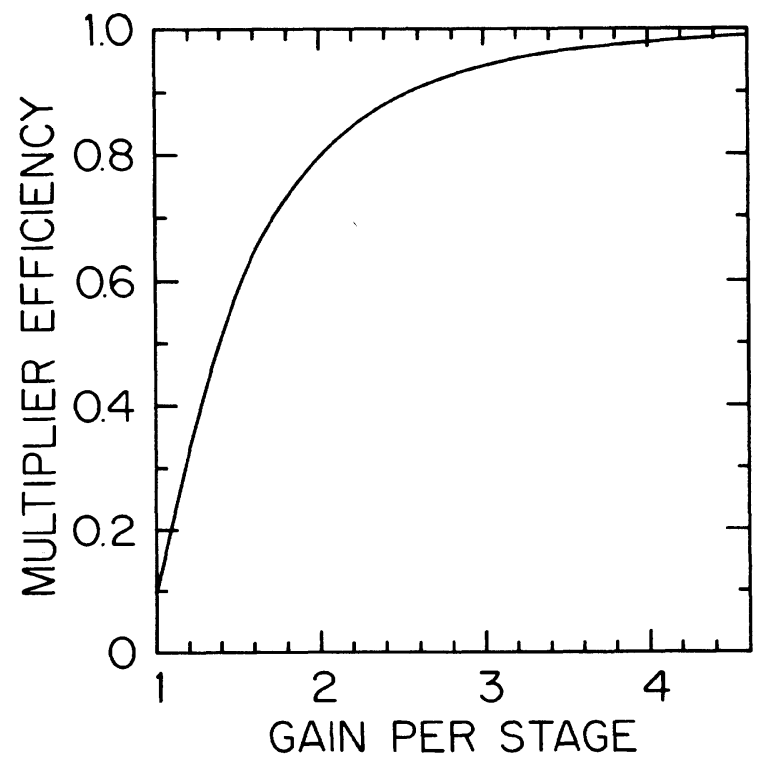

FIG. 13. Detection efficiency vs the average gain per stage calculated for an 18-stage multiplier using Eq. (A3). 
combination used $\left(3.27 \times 10^{13} \mathrm{~V} / \mathrm{C}\right)$, the multiplier gain corresponding to each pulse height was calculated. These values are on the bottom scale. From a measurement of the area under the curve $\left(5.5 \times 10^{9}\right)$ and the number of counts at zero pulse height (14.000), we obtain the average multiplier gain $\left(3.9 \times 10^{5}\right)$. Since it is an 18-stage multiplier, this yields an average gain per stage of 2.05. Referring to the graph in Fig. 13 [obtained from Eq. (A3)], this gain yields an efficiency of 0.81 , the value used in our work.

The PHD calculated from Eq. (A2) for $m=2.05$ is shown as the line in Fig. 13, normalized to the data at a pulse height of $2 \mathrm{~V}$. From the good agreement with the measured values, it appears that the present multiplier obeys Poisson statistics rather well and that therefore this method of determining the efficiency should be valid.

The change in the detection efficiency due to variations in the energy of the electrons incident on the first dynode can be determined from the theory of secondary emission as given by Lye and Dekker. ${ }^{23}$ The first-stage gain as a function of energy $E$ may be obtained from the equation

$$
\begin{aligned}
& \epsilon(E) / \epsilon_{m} \\
& =1.11\left(E_{m} / E\right)^{0.35}\left[1-\exp \left(-2.28 E / E_{m}\right)^{1.35}\right],
\end{aligned}
$$

which was derived from the equations given by them. $E_{m}$ is the energy for which the secondary emission has its maximum value $\epsilon_{m}$. In their development, an important parameter is $n$, the exponent in the energy dependence of the stopping power of the dynode material for electrons. The value of $n$ was taken to be 0.35 as suggested by them.

By this method the variation of the efficiency over the energy range of electrons in this investigation was found to be less than $10 \%$ and therefore we have taken the efficiency to be constant.
*Present address: Institute of Physics, Chinese Academy of Sciences, P.O. Box 603, Beijing, China.

${ }^{1}$ L. H. Toburen, Steven T. Manson, and Yong-Ki Kim, Phys. Rev. A 17, 148 (1978).

${ }^{2}$ J. H. Miller, L. H. Toburen, and Steven T. Manson, Phys. Rev. A 27, 1337 (1983).

${ }^{3}$ W. E. Wilson, J. H. Miller, and L. H. Toburen, J. Chem. Phys. 80, 5631 (1984).

${ }^{4}$ J. B. Crooks and M. E. Rudd, Phys. Rev. A 3, 1628 (1971).

${ }^{5}$ M. E. Rudd, D. Gregoire, and J. B. Crooks, Phys. Rev. A 3, 1635 (1971).

${ }^{6}$ M. E. Rudd and D. H. Madison, Phys. Rev. A 14, 128 (1976).

${ }^{7}$ M. E. Rudd, Radiat. Res. 109, 1 (1987).

${ }^{8}$ M. E. Rudd, Phys. Rev. A 38, 6129 (1988).

${ }^{9}$ M. E. Rudd, L. H. Toburen, and N. Stolterfoht, At. Data Nucl. Data Tables 23, 405 (1979).

${ }^{10}$ M. E. Rudd, Phys. Rev. A 20, 787 (1979).

${ }^{11}$ D. K. Gibson and I. D. Reid, J. Phys. B 19, 3265 (1986).

${ }^{12}$ M. E. Rudd, Y.-K. Kim, D. H. Madison, and J. W. Gallagher,
Rev. Mod. Phys. 57, 965 (1985).

${ }^{13}$ J. Macek, Phys. Rev. A 1, 235 (1970).

${ }^{14}$ Yong-Ki Kim and Mitio Inokuti, Phys. Rev. A 7, 1257 (1973). See also Ref. 3.

${ }^{15}$ See, e.g., Wolfgang Lotz, J. Opt. Soc. Am. 60, 206 (1970).

${ }^{16}$ M. E. Rudd, L. H. Toburen, and N. Stolterfoht, At. Data Nucl. Data Tables 18, 413 (1976).

${ }^{17}$ D. H. Madison, Phys. Rev. A 8, 2449 (1971).

${ }^{18}$ D. H. Madison (private communication).

${ }^{19}$ A. Salin, J. Phys. B 2, 631 (1969).

${ }^{20}$ Francis J. Lombard and Fred Martin, Rev. Sci. Instrum. 32, 200 (1961).

${ }^{21}$ G. C. Baldwin and S. I. Friedman, Rev. Sci. Instrum. 36, 16 (1965).

${ }^{22}$ Robert F. Tusting, Quentin A. Kerns, and Harold K. Knudsen, IRE Trans. Nucl. Sci. NS-9(3), 118 (1962).

${ }^{23}$ Robert G. Lye and A. J. Dekker, Phys. Rev. 107, 977 (1957). See also F. Bordoni, Nucl. Instrum. Methods 97, 405 (1971), but note the error in the equation following Eq. (6). 